\title{
Evaluation of Case Detection of Marijuana-Related Emergency Department Visits
}

\author{
Kathryn H. DeYoung*1, Robert Beum¹, Yushiuan Chen², Moises Maravi', Lourdes W. \\ Yun ${ }^{1}$, Michele Askenazi ${ }^{2}$, Judith Shlay ${ }^{1}$ and Arthur Davidson ${ }^{1}$ \\ ${ }^{1}$ Epidemiology \& Preparedness, Denver Public Health, Denver, CO, USA; ${ }^{2}$ Tri-County Health Department, Greenwood Village, CO, \\ USA
}

\section{Objective}

To evaluate methods of measuring marijuana-related emergency department visits at Denver metropolitan area hospitals participating in the National Syndromic Surveillance Program (NSSP).

\section{Introduction}

NSSP, a Centers for Disease Control and Prevention (CDC) surveillance system, allows timely detection of emergency department (ED) trends by matching chief complaint (CC) text or diagnosis (DX) codes to established syndrome criteria $[1,2]$. No CDC syndrome definition currently exists for marijuana-related visits. Accidental child ingestions and over-consumption of edible products are an emerging concern [3, 4]. A validated marijuana syndrome will allow health departments with access to ED data to measure relative trends and disparities in marijuana-related ED visits.

\section{Methods}

A marijuana syndrome definition which incorporates $\mathrm{CC}$ and $\mathrm{DX}$ variables was developed and evaluated with data from 15 hospitals in Adams, Arapahoe, Denver, and Douglas counties reporting to NSSP. Preliminary marijuana cases were identified based on DX and CC criteria. CC criteria included terms like "marijuana," "canna", and "edible." The DX code variable was searched for International Classification of Disease 9 codes regularly used to identify marijuanarelated cases: 969.6, E854.1, 304.3, and 305.2 (excluding 304.33 and 305.23, which indicate conditions in remission) [5].

The sensitivity of CC and DX criteria were compared by evaluating the percent of preliminary cases matched on CC, DX, or both and examining frequency of matches on specific search terms. Two reviewers then assessed specificity by examining age-stratified case samples ( 20 cases per age group or all cases if there were less than 20) and categorizing the suspected relationship of marijuana to the visit: 1) directly related, 2) incidental, 3) unrelated, and 4) unclear. Findings were used to refine the case criteria and develop a method to exclude or adjust for incidental, unrelated, and unclear cases.

\section{Results}

From January to July 2015, 1717 preliminary marijuana cases (an average of 245 per month) were identified. Of these, $73 \%$ were identified by DX alone, $19 \%$ by CC alone, and $8 \%$ by both CC and DX. The DX code 305.2 ("nondependent cannabis abuse") and CC text "marijuana" and "THC" were the most sensitive, respectively returning 1320, 189, and 113 cases (including ones which matched multiple criteria). Among cases with an age reported, 20-29 year-olds represented the greatest share of cases $(35 \%)$. Cases were detected in all age groups.

Several hospitals were working to consistently report key variables during the evaluation period. Age was missing for 749 cases; CC was missing for 1249 cases; and DX was missing for 333 cases. During the month of August 2015, overall completeness of age improved to $99.5 \%$ and DX code increased to $75.6 \%$. CC completeness continues to improve as hospitals resolve reporting issues.

\section{Conclusions}

The use of CC text and DX code criteria in the marijuana syndrome definition gave sensitivity despite limitations in data completeness. Evaluating cases identified after August 2015 will permit more accurate assessment of the syndrome's specificity. With states around the country exploring or legalizing retail marijuana, a sensitive but specific measurement of temporal and demographic trends in marijuana-related ED visits is needed. Applying a validated marijuana syndrome definition to NSSP data will help monitor emerging trends and inform policies.

\section{Keywords}

Marijuana; Cannabis; Syndromic surveillance

\section{References}

1. Centers for Disease Control and Prevention (CDC). Monitoring health effects of wildfires using the BioSense system-San Diego County, California, October 2007. MMWR Morb Mortal Wkly Rep. 2008 Jul 11;57(27):741-4

2. Benoit SR, Burkom H, McIntyre AF, et al. Pneumonia in US hospitalized patients with influenza-like illness: BioSense, 2007-2010. Epidemiol Infect. 2013 Apr;141(4):805-15. doi: 10.1017/S0950268812001549. Epub 2012 Jul 17.

3. Wang GS, Roosevelt G, Heard K. Pediatric marijuana exposures in a medical marijuana state. JAMA Pediatr. 2013 Jul;167(7):630-3. doi: 10.1001/jamapediatrics.2013.140.

4. Hancock-Allen JB, Barker L, VanDyke M, Holmes DB. Notes from the Field: Death Following Ingestion of an Edible Marijuana Product - Colorado, March 2014. MMWR Morb Mortal Wkly Rep. 2015 Jul 24;64(28):771-2

5. Colorado Department of Public Health and Environment. Monitoring Health Concerns Related to Marijuana in Colorado: 2014. 2015 Mar 4. Available from https://drive.google.com/folderview?id=0BxqXhstk 92DbfnNfSURHd0VFZjEtRFpsVEg3bjM5QUJXOEd0VWZDOUN jSnpWWEFvTVdiUFU\&usp=sharing.

\section{*Kathryn H. DeYoung}

E-mail: kathryn.deyoung@dhha.org 